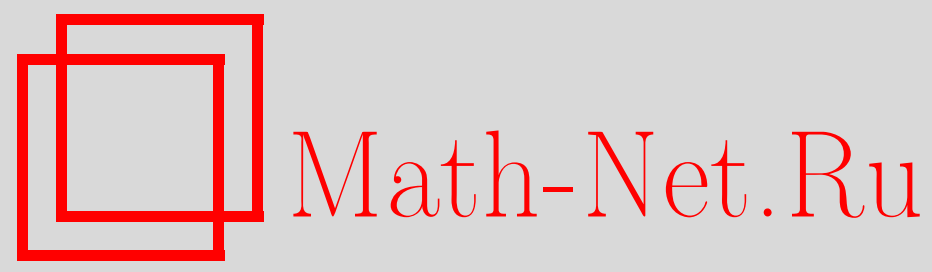

А. А. Бондаренко, Числа классов классических групп, $M a-$ тем. заметки, 2000, том 68, выпуск 1, 49-56

DOI: https://doi.org/10.4213/mzm918

Использование Общероссийского математического портала Math-Net.Ru подразумевает, что вы прочитали и согласны с пользовательским соглашением http://www.mathnet.ru/rus/agreement

Параметры загрузки:

IP : 3.95.254.165

26 апреля 2023 г., 02:15:24

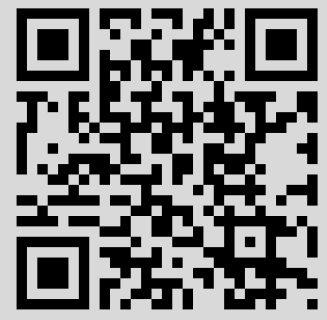




\title{
ЧИСЛА КЛАССОВ КЛАССИЧЕСКИХ ГРУПП
}

\section{А. А. Бондаренко}

\begin{abstract}
В статье завершено описание чисел и групп классов простых классических неопределенных групп над полем алгебраических чисел $K$ в канонических реализациях. Доказан критерий одноклассности односвязной полупростой алгебраической группы в некоторой заданной $K$-реализации. Получен критерий существования одноклассной квадратичной формы среди форм, $\mathbb{Q}$-эквивалентных определенной квадратичной форме от $n \geqslant 3$ переменных над полем рациональных чисел $\mathbb{Q}$.

Библиографоия: 10 названий.
\end{abstract}

Введение. Пусть $G$ - линейная алгебраическая групш, определенная над полем алгебраических чисел $K$. Под заданием реализации группы $G$ будем понимать задание точного $K$-определенного представления $\varphi: G \rightarrow G L_{r}$ и решетки $L(\varphi) \subset K^{r}$. Обозначим через $\varphi(G)_{A}, \varphi(G)_{A(\infty)}$ и $\varphi(G)_{K}$ соответственно групшу аделей, цельх аделей и главных аделей грушшы $\varphi(G)$. Число классов групшы $G$, отвечающее реализации $\varphi$, мы будем обозначать через $\operatorname{cl}(\varphi(G))$ либо $\mathrm{cl}\left(G^{L(\varphi)}\right)$ или просто $\mathrm{cl}(G)$, если это не приводит к недоразумению. Наиболее существенные результаты по проблеме вычисления числа $\mathrm{cl}(G)$ были получены лет двадщать назад (см. [1]) и изложены в монографии [2].

Описанию чисел и групп классов полупростых групп некомпактного типа в наиболее распространенных канонических реализациях посвящена работа [3]. В ней приведены эскизы доказательств теоремы реализации возможных чисел и групп классов на решетках степени $r$ для полупростых $K$-групп $G$ некомпактного типа степени $r$ в случаях, если $G$ - одна из следующих групп:

а) $G$ - полупростая группа с циклической фундаментальной группой, обладающая билинейным симметрическим инвариантом, заданным на пространстве представления;

b) $G$ - присоединенная алгебраическая группа, реализованная в качестве группы автоморфизмов соответствующей алгебры Ли;

c) $G$ - простая классическая группа в каноническом представлении.

Изложение доказательств теоремы реализации в случаях а) и b) почти полностью приведено в [2]. Изложению доказательства теоремы в случае с) посвящен п. 1 этой статьи.

В п. 2 настоящей статьи рассматриваются групшы компактного типа. Основное содержание пункта посвящено исследованию вопроса о существовании одноклассной реализации, когда $G$ - группа компактного типа, т.е. компактна архимедова часть $G_{\infty}$ группы аделей. Для всякой полупростой группы $G$ некомпактного типа всегда существует 
одноклассная реализация группы $G$ и решетку $L(\varphi)$ можно выбрать свободной (см. [2]). Для групш $G$ компактного типа это не так. Более того, это является скорее исключением, чем правилом. Необходимое и достаточное условие одноклассности для односвязных групп в некоторой заданной реализации аннонсировано еще в 1978 году [4]:

TеоремА. Пусть $G$ - односвязная полупростая алгебраическая $K$-группа. Тогда и только тогда $\mathrm{cl}(G)=1$, когда произведение подгрупп $G_{K}$ и $G_{A(\infty)}$ является nодәруппой $G_{A}$.

В п. 2 дано полное доказательство этой теоремы. Кроме того, получен критерий существования одноклассной квадратичной формы среди квадратичных форм, $\mathbb{Q}$-эквивалентных определенной квадратичной форме от $n \geqslant 3$ переменных над полем рациональных чисел $\mathbb{Q}$.

Терминология и обозначения, используемые в настоящей статье, традиционны (см. [2]).

1. Числа классов классических групп некомпактного типа. Описание алгебраических линейных классических групп с практически полными доказательствами приведено в [2]. Как следует из “явного” описания простых классических групп, классические групшы типов $\left(A_{l}\right)$ и $\left(C_{l}\right)$ в канонический реализации односвязны. Мы рассматриваем классические групы некомпактного типа, поэтому описание чисел и груш классов в этом случае тривиально. Классические группы типа $\left(B_{l}\right)$ - это специальные ортогональные групш, типа $\left(D_{l}\right)$ - специальные ортогональные групы и специальные унитарные группы. Описание чисел классов ортогональных групп неопределенных квадратичных форм от $n \geqslant 3$ переменных получено Кнезером и изложено в [2].

Таким образом, для завершения описания чисел и групп классов простьх классических групп остается это сделать для специальных унитарных групп $S U_{l}(D, h)$, где $D-$ тело кватернионов над $K$ с инволюцией первого рода $\sigma$, а невырожденная форма $h$ эрмитова, если $\sigma$ имеет первьй тип, и косоэрмитова, если $\sigma$ имеет второй тип.

Для определенности будем считать, что форма $h$ косоэрмитова и инволюция $\sigma-$ каноническая инволюция сопряжения (она второго типа).

На самом деле специальная унитарная группа естественным образом отождествляется с группой рациональных точек алгебраической группы (см. [2]). Именно эту алгебраическую группу $G$ мы будем иметь в виду, говоря о специальной унитарной группе как об алгебраической группе. Фундаментальная группа этой алгебраической групшы второго порядка. Описание групп и чисел классов в этом случае дает

Теорема 1. Пусть $G$ - K-определенная специальная унитарная әруппа косоәрмитовой формы $h$ размерности $l$ над телом кватернионов над $K$ к канонической инволючией сопряжения. Тогда для любой конечной абелевой группь $B$ әкпоненты 2 существует решетка $L_{B} \subset K^{4 l}$ такая, что $\mathscr{G} \operatorname{cl}\left(G^{L_{B}}\right) \cong B$. В частности, для любого числа вида $2^{d}$ существует решетка $L(d) \subset K^{4 l}$ такая, что $\operatorname{cl}\left(G^{L(d)}\right)=2^{d}$.

ЗАмЕчАниЕ. Описание групп и чисел классов унитарной групшы приведено в теореме 3 из [3], но форма $h$ взята эрмитова, поэтому инволюция $\sigma$ тела кватернионов первого типа, а не просто первого рода, как это можно понять из [3].

Способ доказательства этой теоремы является модификацией общего метода описания чисел и групш классов полупростых алгебраических групп, изложенного в деталях 
в монографии [2]. Но для доказательства этой теоремы необходимо иметь локальную решетку с достаточно "малым" стабилизатором для бесконечного числа нормирований из $V_{f}^{K}$. Описание необходимой решетки в пространстве $K_{v}^{4 l}$ приведено ниже. Идея построения этой решетки подобна идее построения решетки в случае а).

Опишем алгебраическую "реализацию” специальной унитарной группы $S U_{l}(D, h)$ в подходящем виде. Пусть $E-l$-мерное векторное пространство над телом $D$ с центром $K, h$-невырожденная косоэрмитова форма на $E \times E, \sigma$-каноническая инволюция тела кватернионов $D$. В пространстве $D^{l}$ существует базис $u_{1}, \ldots, u_{l}$ (см. [2], [5]) ортогональный относительно формы $h$, т.е. $h\left(u_{i}, u_{j}\right)=0$ при $i \neq j, h\left(u_{i}, u_{i}\right)=a_{i} \in D$ и $\sigma\left(a_{i}\right)=-a_{i}$. Тогда в базисе $u_{1}, \ldots, u_{l}$ группа $S U_{l}(D, h)=\left\{A \in M_{l}(D) /{ }^{*} A H A=H\right.$, $\left.N r d_{M_{l}(D) / K}(A)=1\right\}$, где матрища $H=\operatorname{diag}\left(a_{1}, a_{2}, \ldots, a_{l}\right),{ }^{*} A=\left(\sigma\left(a^{\beta \alpha}\right)\right)$, если $A=\left(a^{\alpha \beta}\right)$.

Если $x=\left(x^{\alpha \beta}\right) \in M_{l}(D)$, то при регулярном представлении $\rho: D \rightarrow M_{4}(K)$ тела $D$ над $K$ матрища $x$ переходит в матрицу $\left(x_{i j}^{\alpha \beta}\right) \in M_{4 l}(K)(i, j=1,2,3,4 ; \alpha, \beta=1,2, \ldots, l)$, где $\left(x_{i j}^{\alpha \beta}\right)(i, j=1,2,3,4)$ - регулярное представление элемента $x^{\alpha \beta} \in D$ матрицей над $K$ (см. [2]). При таком соответствии множество матриц из $S U_{l}(D, h)$ переходит в множество $K$-точек некоторой алгебраической $K$-групшы $G$, т.е. $S U(D, h)$ реализуется как группа автоморфизмов пространства $K^{4 l}$ в некотором базисе

$$
e_{1}, e_{2}, \ldots, e_{4 l}
$$

В пространстве $K_{v}^{4 l}$ построим необходимую решетку для бесконечного множества нормирований $v \in V_{f}^{K}$. Элементы матрищы из образа $S U_{l}(D, H)$ в $M_{4 l}(K)$ при гомоморфизме, индуцируемом $\rho$, удовлетворяют некоторой системе линейных уравнений, определяющей $\rho(D)$ как подпространство в $M_{4}(K)$ :

$$
f_{k}\left(x_{i j}^{\alpha \beta}\right)=0, \quad i, j=1,2,3,4, \quad k=1, \ldots, s,
$$

для всех $\alpha, \beta=1, \ldots, l$, и матричному уравнению

$$
\left(\widetilde{\sigma}\left(x_{i j}^{\beta \alpha}\right)\right) \widetilde{H}\left(x_{i j}^{\alpha \beta}\right)=\widetilde{H},
$$

где $\widetilde{H}=\operatorname{diag}\left(H_{1}, H_{2}, \ldots, H_{l}\right)$ - образ $H$ при регулярном представлении $\rho$ элементов матрицы $H=\operatorname{diag}\left(a_{1}, a_{2}, \ldots, a_{l}\right), \widetilde{\sigma}$ - линейный автоморфизм $M_{4}(K)$, продолжающий инволюцию $\rho \sigma \rho^{-1}$ на $\rho(D)$. По определению $G$ этим уравнениям удовлетворяют и элементы всех матриц из группы $G$, реализованной в базисе (1).

Пусть теперь $L_{v}-O_{v}$-решетка пространства $K_{v}^{4 l}$ с базисом

$$
e_{1}, \pi e_{2}, \ldots, \pi_{v}^{4 l-1} e_{4 l},
$$

где $\pi_{v}$ - униформизуюший элемент относительно нормирования $v \in \in V_{f}^{K}$. Опишем стабилизатор $G_{O v}^{L_{v}}$ решетки $L_{v}$ в $G_{K v}$.

Если $g \in G_{O v}^{L_{v}}$ и $g=\left(g_{r t}\right)$ в базисе $(1)$, то

$$
g\left(\pi_{v}^{t-1} e_{t}\right)=\sum_{r=1}^{4 l} \pi_{v}^{t-r} g_{r t} \cdot\left(\pi^{r-1} e_{r}\right)
$$


для всех $t=1,2, \ldots, 4 l$. Поэтому $g_{r t} \in \mathscr{P}_{v}^{r-t}$ при $r \geqslant t$, т.е. все элементы в матрице $g$ ниже главной диагонали сравнимы с нулем по $\bmod \pi_{v}$. Запишем матрицу $g$ в блочном виде. Пусть $g=\left(a_{i j}^{\alpha \beta}\right)(i, j=1,2,3,4 ; \alpha, \beta=1,2, \ldots, l)$. Рассмотрим матрицу $B=\left(\widetilde{\sigma}\left(a_{i j}^{\beta \alpha}\right)\right)$ $(i, j=1,2,3,4 ; \alpha, \beta=1,2, \ldots, l)$. Каждый блок $\left(a_{i j}^{\alpha \beta}\right)(i, j=1,2,3,4)$ матрицы $g$ удовлетворяет уравнениям (2), поэтому имеет вид

$$
\left(\begin{array}{cccc}
a_{0} & a_{1} \beta_{1} & a_{2} \beta_{2} & -a_{3} \beta_{1} \beta_{2} \\
a_{1} & a_{0} & a_{3} \beta_{2} & -a_{2} \beta_{2} \\
a_{2} & -a_{3} \beta_{1} & a_{0} & a_{1} \beta_{1} \\
a_{3} & -a_{2} & a_{1} & a_{0}
\end{array}\right),
$$

где $\beta_{2}, \beta_{1} \in K$-постоянные, с помощью которых задается умножение кватернионов в $D$ (см., например, [5]), $a_{0}, a_{1}, a_{2}, a_{3}$ - элементы поля $K_{v}$. Учитьвая действие $\widetilde{\sigma}$, получаем, что блок $\widetilde{\sigma}\left(a_{i j}^{\alpha \beta}\right)(i, j=1,2,3,4)$ имеет вид

$$
\left(\begin{array}{cccc}
a_{0} & -a_{1} \beta_{1} & -a_{2} \beta_{2} & a_{3} \beta_{1} \beta_{2} \\
-a_{1} & a_{0} & -a_{3} \beta_{3} & a_{2} \beta_{2} \\
-a_{2} & a_{3} \beta_{1} & a_{0} & -a_{1} \beta_{1} \\
-a_{3} & a_{2} & -a_{1} & a_{0}
\end{array}\right) .
$$

Поэтому все элементы блоков матрицы $B$ выше блоков главной диагонали сравнимы с нулем по $\bmod \pi_{v}$. Матрица $g$ удовлетворяет уравнению $(3)$; значит, $B=\widetilde{H} g^{-1} \widetilde{H}^{-1}$. Но $g^{-1} \in G_{O_{v}}^{L_{v}}$; следовательно, все элементы матрицы $g^{-1}$ ниже главной диагонали сравнимы с нулем по $\bmod \pi_{v} ; \widetilde{H}=\operatorname{diag}\left(H_{1}, \ldots, H_{l}\right)$, поэтому во всех блоках матрищы $B$ ниже блоков главной диагонали элементы сравнимы с нулем по $\bmod \pi_{v}$ почти для всех $v \in V_{f}^{K}$ (достаточно исключить нормирования $v \in S_{1}$, для которых $\widetilde{H}$ либо $\widetilde{H}^{-1}$ имеют элементы, не являющиеся целыми $v$-адическими числами). Следовательно, все элементы матрищы $B$ вне диагональных блоков сравнимы с нулем по $\bmod \pi_{v}$ почти для всех $v \in V_{f}^{K}$. Сравнивая матрицы $g$ и $B$, замечаем, что и все элементы матрицы $g$ вне блоков главной диагонали сравнимы с нулем по $\bmod \pi_{v}$ почти для всех $v \in V_{f}^{K}$.

Осталось рассмотреть диагональные блоки $\left(a_{i j}^{\alpha \alpha}\right)(i, j=1,2,3,4)$ матрищы $g$. Все элементы диагонального блока ниже главной диагонали сравнимы с нулем по $\bmod \pi_{v}$, ибо таковы все элементы матрицы $g$ ниже главной диагонали. Поэтому, учитьвая симметрию блока (см. вид блока), получаем, что все элементы диагонального блока выше главной диагонали сравнимы с нулем по $\bmod \pi_{v}$ почти для всех $v \in V_{f}^{K}$ (достаточно исключить $v \in S_{2}$, когда $\beta_{1}$ либо $\beta_{2}$ не являются целыми $v$-адическими числами). Следовательно, диагональные блоки $\left(a_{i j}^{\alpha \alpha}\right)$ и $\widetilde{\sigma}\left(a_{i j}^{\alpha \alpha}\right)$ сравнимы с матрищей вида

$$
\left(\begin{array}{cccc}
a_{0}^{\alpha \alpha} & 0 & 0 & 0 \\
0 & a_{0}^{\alpha \alpha} & 0 & 0 \\
0 & 0 & a_{0}^{\alpha \alpha} & 0 \\
0 & 0 & 0 & a_{0}^{\alpha \alpha}
\end{array}\right)
$$

по $\bmod \pi_{v}$ для всех $v \in V_{f}^{K} \backslash S_{2}$.

Элементы всех блоков матриц $g$ и $B$, за исключением диагональньх, сравнимы с нулем по $\bmod \pi_{v}$ для всех $v \in V_{f}^{K} \backslash S_{1}$. Поэтому почти для всех $v \in V_{f}^{K}$ для каждого диагонального блока из равенства (3) получаем матричное сравнение

$$
\tilde{\sigma}\left(a_{i j}^{\alpha \alpha}\right) H_{\alpha}\left(a_{i j}^{\alpha \alpha}\right) \equiv H_{\alpha}\left(\bmod \pi_{v}\right)
$$


где $H_{\alpha}$ - матрища, соответствующая регулярному представлению элемента $a_{\alpha}$ матрицы $H$. Исключив из $V_{f}^{K}$, возможно, еще конечное число нормирований $v$, при которых некоторые $H_{\alpha}$ сравнимы с нулевой матрищей по $\bmod \pi_{v}$, получим из $(5)$, что $\left(a_{0}^{\alpha \alpha}\right)^{2} \equiv 1$ $\left(\bmod \pi_{v}\right)$ почти для всех $v \in V_{f}^{K}$. Поэтому $a_{0}^{\alpha \alpha} \equiv \pm 1\left(\bmod \pi_{v}\right)$.

Подводя итог, получаем, что $G_{O v}^{L_{v}} \subset \Gamma G_{O_{v}}\left(\mathscr{P}_{v}\right)$, где $G_{O_{v}}\left(\mathscr{P}_{v}\right)$-конгруэнц-подгрупша в $G_{O_{v}}^{L_{v}}$, отвечающая максимальному идеалу $\mathscr{P}_{v}$ кольца $O_{v}, \Gamma=\left\{\left(e_{i j}\right), e_{i i}= \pm 1, i=1\right.$, $2, \ldots, 4 l ; e_{i j}=0$ при $\left.i \neq j\right\}$, почти для всех $v \in V_{f}^{K}$. Иначе, справедлива

ЛЕмма. Пусть $G$ - K-определенная спечиальная унитарная группа косоэрмитовой формы $h$ размерности $l$ над телом кватернионов над $K$ с канонической инволюиией сопряэсения. Тогда существует конечное множество неархимедовых нормирований $S$ таких, что для любого $v \in V_{f}^{K} \backslash S$ существует решетка $L_{v} \subset K_{v}^{4 l}$, для которой $G_{O_{v}}^{L_{v}} \subset \Gamma G_{O_{v}}\left(\mathscr{P}_{v}\right)$, әде $\Gamma=\left\{\left(e_{i j}\right), e_{i i}= \pm 1, i=1,2, \ldots, 4 l ; e_{i j}=0\right.$ npu $i \neq j\}$.

Лемма дает необходимую локальную решетку $L_{v}$ в пространстве $K_{v}^{4 l}$. После этого доказательство теоремы 1 проводится по отработанной схеме (см., например, [2]).

2. Числа классов групп компактного типа. Наиболее законченные результаты по описанию чисел классов групп компактного типа, известные в настоящее время, изложены в [2]. После знакомства с ними возникает закономерньй вопрос: какое наименьшее значение принимает число $\operatorname{cl}(\varphi(G))$ при возможных $K$-реализациях $\varphi$ групшы $G$ ? В частности, возникает естественная задача определения условий, которым должна удовлетворять группа $G$, чтобы для нее существовала одноклассная реализация.

Критерий одноклассности односвязной полупростой алгебраической грушы в некоторой заданной реализации устанавливает

ТЕОРема 2. Пусть $G$-односвязная полупростая алгебраическая $K$-группа. Тогда и только тогда $\mathrm{cl}(G)=1$, когда произведение подгрупп $G_{K} u G_{A(\infty)}$ является nодгруппой $G_{A}$.

ДокАЗАТЕЛЬСТво. Необходимость условия теоремы очевидна.

Докажем достаточность. Для $v_{0} \in V_{f}^{K}, \alpha \in G_{K_{v_{0}}}$ обозначим через $x^{v_{0}}(\alpha)$ адель с компонентами

$$
x_{v}= \begin{cases}1, & v \neq v_{0}, \\ \alpha, & v=v_{0} .\end{cases}
$$

Пусть $v_{0} \in V_{f}^{K}$ такое, что $G_{K_{v_{0}}}$ - некомпактная группа (такое нормирование существует). А тог да из сильной апроксимационной теоремы (см. [2]) имеем $G_{K} G_{A\left(\infty, v_{0}\right)}=G_{A}$, где

$$
G_{A\left(\infty, v_{0}\right)}=\prod_{v \in\left(V_{\infty}^{K}, v_{0}\right)} G_{K_{v}} \cdot \prod_{v \in V_{f}^{K} \backslash v_{0}} G_{O_{v}} .
$$

Поэтому любой адель $g=\left(g_{v}\right) \in G_{A}$ представляется в виде $g=g_{1} x^{v_{0}}(\alpha) g_{2}$, где $g_{1} \in G_{K}, g_{2} \in G_{A(\infty)}$ и $\alpha-$ подходящий элемент из $G_{K v_{0}}$. Так как $G_{K} G_{A(\infty)}-$ группа, для доказательства включения $G_{A} \subset G_{K} G_{A(\infty)}$ достаточно установить, что $x^{v_{0}}(\alpha) \in G_{K} G_{A(\infty)}$ для любого $\alpha \in G_{K_{v_{0}}}$.

Из теоремы о конечности числа классов [2] следует, что индекс $\left[G_{A}: G_{K} G_{A(\infty)}\right]$ конечен. Пусть $u$ - унипотентньй элемент в $G_{K_{v_{0}}}, U$ - содержащая его однопараметрическая подгруппа. Тогда $x^{v_{0}}\left(U_{K_{v_{0}}}\right)$ является полной абелевой группой, поэтому 
$x^{v_{0}}\left(U_{v_{0}}\right) \subset G_{K} G_{A(\infty)}$. Так как $G_{K_{v_{0}}}-$ некомпактная групша, из справедливости для $G_{K_{v_{0}}}$ гипотезы Кнезера-Титса (см. [2]) следует, что $G_{K_{v_{0}}}$ порождается унипотентными элементами из $G_{K_{v_{0}}}$. С учетом отмеченного выше факта это доказьвает включение $x^{v_{0}}(\alpha) \in G_{K} G_{A(\infty)}$ для любого $\alpha \in G_{K_{v_{0}}}$. Теорема доказана.

ЗАмЕчаниЕ 1 . Пусть $G$-полупростая алгебраическая $K$-группа, $\pi: G \rightarrow \widetilde{G}$ - универсальное $K$-определенное накрытие, $f$ - показатель ядра этого накрытия.

Совершенно аналогично доказательству теоремы 2 доказьвается, что $G_{K} G_{A(\infty)}-$ подгруппа $G_{A}$ тогда и только тогда, когда $G_{K} G_{A(\infty)} \supset \pi_{A}\left(\widetilde{G}_{A}\right)$. Это утверждение похожим образом установлено при доказательстве теоремы 2 из [6].

ЗАмЕчАниЕ 2. Возникает естественный вопрос: существуют ли полупростые группы компактного типа, для которых $G_{K} G_{A(\infty)}$ может быть группой? Существование полупростых групш $G$ компакного типа и их реализаций $\varphi$ таких, что $\varphi(G)_{K} \varphi(G)_{A(\infty)}-$ группа, следует из известных результатов по описанию одноклассных положительно определенных квадратичных форм размерности $n \geqslant 3$ над полем рациональных чисел (см., например, [7], [8]). В частности, отсюда следует существование односвязных простых $K$-групп компактного типа и их реализаций $\varphi$ таких, что $\varphi(G)_{K} \varphi(G)_{A(\infty)}-$ группа. Поэтому теорема 2 и утверждения из замечания 1 являются не просто абстракными утверждениями, а имеют реальное основание для применения.

Отметим факт, которьй показьвает, что существование такой $K$-реализации $G$, что $G_{K} G_{A(\infty)}$ - подгрупш $G_{A}$, определяется внутренними свойствами самой группы и не зависит от выбора точного представления.

ПРЕДЛОЖЕНИЕ. Пусть $G \subset G L_{n}$ - произвольная алгебраическая $K$-группа степени $n$. Предположим, что существует решетка $L \subset K^{n}$ такая, что $G_{K} G_{A(\infty)}^{L}$ - подгруппа $G_{A}$. Тогда для любого точного $K$-определенного представления $\varphi: G \rightarrow G L_{r}$ также существует решетка $L(\varphi) \subset K^{r}$ такая, что $\varphi(G)_{K} \varphi(G)_{A(\infty)}^{L(\varphi)}-$ noдəpynna $\varphi(G)_{A}$.

ДокАЗАТЕЛЬСтво. Обозначим через $S$ конечное подмножество $V_{f}^{K}$ такое, что для $v \in V_{f}^{K} \backslash S$ выполняются следующие условия: $L_{v}=O_{v}^{n}$ и морфизм $\varphi$ определен над $O_{v}$. Для каждого $v \in S$ группа $\varphi\left(G_{O_{v}}^{L_{v}}\right)$ компактна, поэтому существует решетка $M_{v} \subset K_{v}^{r}$ такая, что $\varphi\left(G_{O_{v}}^{L_{v}}\right) \subset \varphi\left(G_{O_{v}}\right)^{M_{v}}$. Зададим локализации искомой решетки $L(\varphi)$ следующим образом:

$$
L(\varphi)_{v}= \begin{cases}M_{v}, & v \in S, \\ O_{v}^{r}, & v \notin S .\end{cases}
$$

Тогда из наших построений вытекает, что $\varphi\left(G_{A(\infty)}^{L}\right) \subset \varphi(G)_{A(\infty)}^{L(\varphi)}$. Поэтому $\varphi(G)_{K} \times$ $\varphi(G)_{A(\infty)}^{L(\varphi)} \supset \varphi\left(G_{K} G_{A(\infty)}^{L}\right)$. Но $G_{K} G_{A(\infty)}^{L}$ - подгруппа $G_{A}$; следовательно, по утверждению из замечания $1 G_{K} G_{A(\infty)}^{L} \supset \pi_{A}\left(\widetilde{G}_{A}\right)$. А тогда $\varphi(G)_{K} \varphi(G)_{A(\infty)}^{L(\varphi)}$ - подгруппа $\varphi(G)_{A}$. Предложение доказано.

Особый интерес представляет вопрос о существовании одноклассной квадратичной формы $f$ над вполне вещественным полем $K$ среди квадратичных форм, $K$-эквивалентных положительно определенной форме $g$ от $n \geqslant 3$ переменных. Эта задача над полем рациональных чисел рассматривалась многими авторами (см. [7], [8]). 
Получим критерий существования одноклассной квадратичной формы среди $\mathbb{Q}-э к-$ вивалентных квадратичных форм положительно определенной квадратичной форме $g$ налд $\mathbb{Q}$.

Хорошо известно (см. [2]), что $\operatorname{cl}(H)=c(g)$, где $H=O(g), c(g)$ - число классов в роде формы $g$. Поэтому существование одноклассной квадратичной формы $f$ среди эквивалентных $g$ равносильно существованию одноклассной реализации групшы $H=O(g)$.

Пусть $g$ - положительно определенная квадратичная форма от $n \geqslant 3$ переменных над кольцом $\mathbb{Z}$ целых рациональных чисел, $H=O(g)$ и $G=S O(g)$. Определенность квадратичной формы $g$ равносильна тому, что $G_{\infty}=G_{R}$ компактна.

Имеет место

Теорема 3. Пусть $g$ - положительно определенная квадратичная форма степени $n \geqslant 3$ над $\mathbb{Z}$. Одноклассная квадратичная форма $f, \mathbb{Q}$-әквивалентная $g$, существует тогда и только тогда, когда $G_{\mathbb{Q}} G_{A(\infty)}^{L}$ является подгруппой $G_{A} \boldsymbol{\theta}$ некоторой $\mathbb{Q}$-реализации группы $G$.

ДокаЗАтЕльство. Достаточность. Пусть $G_{\mathbb{Q}} G_{A(\infty)}^{L}$ является подгруппой $G_{A}$ в некоторой $\mathbb{Q}$-реализации группы $G$. Согласно предложению можем считать, что $G=S O(g)$ и решетка $L \subset \mathbb{Q}^{n}$. Хорошо известно, что множество $V_{f}^{\mathbb{Q}}$ исчерпывается $p$-адическими нормированиями. $G_{\mathbb{Z}_{p}}^{L_{p}}$-максимальная компактная подгруппа $G_{\mathbb{Q}_{p}}$ для всех $p$-адических нормирований, за исключением, возможно, конечного множества нормирований $S \subset V_{f}^{\mathbb{Q}}$ (см. [2]). Для $p \in S$ группа $G_{\mathbb{Z}_{p}}^{L_{p}}$ содержится в некоторой максимальной компактной подгруппе $G_{\mathbb{Z}_{p}}^{M_{p}}$, где $M_{p}$ - решетка из $\mathbb{Q}_{p}^{n}$. Пусть $M$ - решетка из $\mathbb{Q}^{n}$ с локализациями

$$
M_{p}= \begin{cases}L_{p}, & p \notin S, \\ M_{p}, & p \in S .\end{cases}
$$

Очевидно, что $G_{A(\infty)}^{L} \subset G_{A(\infty)}^{M}$, и следовательно, $G_{\mathbb{Q}} G_{A(\infty)}^{M}-$ подгруппа $G_{A}$. Известно (см., например, [9], [10]), что $\Theta\left(G_{\mathbb{Z}_{p}}^{M_{p}}\right) \supset \mathbb{Z}_{p}^{*} \mathbb{Q}_{p}^{* 2}$ для любого $p$, где $\Theta$ - спинорное отображение на $G$.

Образ $\Theta\left(G_{\mathbb{Q}}\right)=\left\{\alpha \mathbb{Q}^{* 2} / \alpha \in \mathbb{Q}, \alpha>0\right\}$ (см. [5]) и, значит, $\Theta\left(G_{\mathbb{Q}}\right) \supset\left\{p \mathbb{Q}^{* 2} / p>0-\right.$ произвольное простое число\}. Следовательно, $\Theta\left(G_{\mathbb{Q}}\right) \Theta\left(G_{A(\infty)}^{M}\right)=\Theta\left(G_{A}\right)$. Как следует из замечания $1, G_{\mathbb{Q}} G_{A(\infty)}^{M}$ содержит $\pi_{A}\left(\widetilde{G}_{A}\right)$ и поэтому $\operatorname{cl}\left(G^{M}\right)=1$. А тем более $\operatorname{cl}\left(O(g)^{M}\right)=1$. Через $f$ обозначим реализацию квадратичной формы $g$ в базисе решетки $M$ ( $M$ - свободная решетка, ибо такова произвольная решетка над $\mathbb{Z})$. Очевидно, $f$ и есть одноклассная квадратичная форма, $\mathbb{Q}$-эквивалентная $g$.

Необходимость. Пусть $f$ - одноклассная квадратичная форма, $\mathbb{Q}$-эквивалентная $g$. Тогда $M=\mathbb{Z}^{n}$ - одноклассная решетка относительно действия группы $H=O(f)$. Хорошо известно (см. [2]), что $\operatorname{cl}\left(H^{M}\right)=\operatorname{cl}\left(G^{M}\right)$, если квадратичная решетка $M$ имеет автоморфизм с определителем -1 . Если $n$ нечетно, то так всегда. И в этом случае $G_{K} G_{A(\infty)}^{M}=G_{A}$, т.е. $G_{K} G_{A(\infty)}^{M}-$ групша. Остается рассмотреть случай четного $n$.

Можем считать, что $H_{\mathbb{Z}_{p}}^{M_{p}}$ - максимальная компактная подгруппа $H_{\mathbb{Q}_{p}}$ для любого $p$. Не ограничивая общности (см. [10]) можем считать, что $M_{p}=\mathbb{Z}_{p}\left[p e_{1}^{(p)}, \ldots, p e_{s}^{(p)}, e_{s+1}^{(p)}\right.$, $\left.\ldots, e_{n}^{(p)}\right], 0 \leqslant s \leqslant l \leqslant n / 2$, где $l$ - индекс квадратичной формы $f$ над $\mathbb{Q}_{p}, e_{1}^{(p)}, \ldots, e_{n}^{(p)}-$ 
базис Витта квадратичного пространства $\mathbb{Q}_{p}^{n}$, выбранного так, что $\mathbb{Z}_{p}\left[e_{l+1}^{(p)}, \ldots, e_{n-l}^{(p)}\right]-$ $2 \mathbb{Z}_{p}$-максимальная решетка в соответствуюшем пространстве.

Пусть $S$ - множество $p$-адических нормирований, для которых $s=l=n / 2$. Положим $b$ равным произведению простых чисел, соответствующих нормированиям из $S$. Рассмотрим квадратичную форму $\varphi=(1 / b) f$. Заметим, что $\varphi \mathbb{Z}_{p}$-эквивалентна квадратичной форме с цельми $p$-адическими коэффициентами для всех $p$. Следовательно, $\varphi$ с коэффищентами из $\mathbb{Z}$. Далее, так как $\mathbb{Z}_{p}$-эквивалентные формы представляют над $\mathbb{Z}_{p}$ одно и то же множество чисел, то $\varphi$ представляет 2 над всеми $\mathbb{Z}_{p}$. Очевидно, $H=O(f)$ $=O(\varphi)$ и $\varphi$-одноклассная квадратичная форма.

Напомним утверждение, применяемое многими авторами (в частности, в [7], [8]): пусть $\varphi$ - одноклассная положительно определенная квадратичная форма над $\mathbb{Z}, a-$ целое положительное число; $\varphi$ представляет $a$ над $\mathbb{Z}$ тогда и только тогда, когда $\varphi$ представляет $a$ над $\mathbb{Z}_{p}$ для всех $p$. Отсюда следует, что $\varphi$ представляет 2 над $\mathbb{Z}$, т.е. существует $u \in M$ такое, что $\varphi(u)=2$. Определитель преобразования решетки $M$

$$
\tau_{u}(x)=x-\frac{2 B(u, x)}{\varphi(u)} u=x-B(u, x) u,
$$

где $B$ - билинейная форма, соответствующая квадратичной форме $\varphi$, равен 1 . Следовательно, $\operatorname{cl}\left(G^{M}\right)=\operatorname{cl}\left(H^{M}\right)=1$, а значит, $G_{\mathbb{Q}} G_{A(\infty)}^{M}-$ группа. Это и завершает доказательство теоремы.

\section{СПИСОК ЦИТИРОВАННОЙ ЛИТЕРАТУРЫ}

[1] Платонов В.П., Рапинчук А. С. Алгебраические группы // Итоги науки и техн. Алгебра. Топология. Геометрия. Т. 21. М.: ВИНИТИ, 1983. С. 80-134.

[2] Платонов В.П., Рапинчук А. С. Алгебраические группы и теория чисел. М.: Наука, 1991.

[3] Бондаренко А. А. Числа и группы классов полупростых неопределенных алгебраических групп в канонических реализациях // Докл. АН БССР. 1981. Т. 25. № 3. С. 773-776.

[4] Бондаренко А. А. О числе классов групп аделей алгебраических групп // VI Всесоюзньй симпозиум по теории групп. Тезисы докл. Киев, 1978. С. 10-11.

[5] Дьедонне ЖК. Геометрия классических групп. М.: Мир, 1974.

[6] Платонов В. П., Бондаренко А. А., Рапинчук А. С. Числа и группы классов алгебраических групп. II // Изв. АН СССР. Сер. матем. 1980. Т. 44. № 2. С. 395-414.

[7] Watson G. L. One-class genera of positive ternary quadratic forms // Math. 1975. V. 22. № 1. P. 1-11.

[8] Watson G. L. One-class genera of positive quadratic forms in at least five variables // Acta Arith. 1975. V. 26. №3. P. 309-327.

[9] Бондаренко А. А. Классификация максимальных арифметических подгрупп неопределенных ортогональых групп типа $\left(B_{l}\right)$ // Матем. сб. 1985. Т. $127(169)$. № 1. С. 72-91.

[10] Бондаренко А. А. К проблеме максимальности арифметических подгрупп неопределенных ортогональных групп типа $\left(D_{l}\right)$ // Матем. сб. 1990. Т. 181. №3. С. 388-401.

Белорусский государственный университет, г. Минск

Поступило 03.09.1998 